\title{
Pressure to work through periods of short term sickness
}

\author{
Can have long term negative effects on health and productivity
}

\author{
Kevin Dew professor of sociology \\ School of Social and Cultural Studies, Victoria University of Wellington, Wellington 6140, New Zealand/Aotearoa
}

Recent research shows that during a period of four weeks a third of doctors and nurses have worked when they should have taken sick leave, and that, on average, this nearly halves their working capacity. ${ }^{1}$ Presenteeism is the phenomenon of workers turning up to work despite medical conditions that should prevent them from attending. ${ }^{2}$ Although it has been associated with negative health effects and with loss of productivity, it is only in recent years that presenteeism has gained some focus in research. ${ }^{3}$ In July 2003 a word search for absenteeism in Web of Science produced 1262 hits, whereas presenteeism produced only 11 hits. ${ }^{4}$ The same search in May 2011 scored 3388 hits for absenteeism and 287 for presenteeism.

Around $26 \%$ of healthcare workers report presenteeism in the past seven days and about $85 \%$ of general practitioners and hospital workers report being sick at work some time. ${ }^{5}$ Research has identified many risk factors for presenteeism, and much more work is needed to determine what factors are modifiable and what sort of impact successful modifications would have.

Presenteeism is more strongly noted in certain occupations, particularly those that involve caring and teaching. ${ }^{6}$ For health professionals hospital culture plays a role, ${ }^{7}$ and variations among health professionals reporting presenteeism suggest that it is higher for those who have greater responsibility for patient care. ${ }^{7}$ Similarly, workers who lack back-up and for whom work accumulates while they are absent are more inclined to practise presenteeism. ${ }^{3}$ Other work related factors that foster presenteeism include working in teams where expectations to be present are high, ${ }^{3}$ limited sick leave entitlements, ${ }^{8}$ high job demands (which also correlates with burnout), ${ }^{9}$ low levels of job satisfaction, ${ }^{1}$ fear of dismissal, and concerns about promotion opportunities. ${ }^{9}$ But stimulating work environments can also promote presenteeism. ${ }^{9}$ Factors external to the workplace include financial problems and high unemployment rates. ${ }^{6}$ Female workers may practise presenteeism more, ${ }^{6}$ particularly as they are more likely to be in caring and teaching occupations, but in some occupations women resist the pressure to work when sick more than men do. ${ }^{3}$

Certain medical conditions are likely to predispose to presenteeism. Psychological problems, particularly depression, may do so because they are not seen as legitimate reasons for absence. ${ }^{3}$ Migraine is also associated with presenteeism, ${ }^{3}$ and the phenomenon is also more common in workers with poorer health, probably because of concerns about finding other employment in the event of redundancy. ${ }^{6}$

Certain individual traits, such as difficulty in resisting other people's wishes, have also been associated with presenteeism. ${ }^{6}$ Presenteeism may be higher in people whose self esteem is dependent on their individual performance_-for example, young adults. ${ }^{10}$

Presenteeism increases morbidity,${ }^{85}$ including musculoskeletal pain, fatigue, depression, ${ }^{2}$ and serious coronary events. ${ }^{11}$ It leads to exhaustion, and in a spiralling fashion exhaustion leads to more presenteeism. ${ }^{9}$

In healthcare settings presenteeism poses a particular concern because of the spread of infections, ${ }^{5}$ so preventive measures have been instituted. Less attention has been paid to presenteeism and its prevention in other occupations, however. Presenteeism should be taken seriously if we are concerned with occupational health or workplace productivity. Presenteeism is a complex phenomenon that needs to be approached from several different levels including workplace culture, workplace policies, and carefully considered interventions from health practitioners.

Work related factors have a bigger effect on presenteeism than personal circumstances, although these are still important. ${ }^{1}$ Health promotion at the workplace could emphasise the control that workers have over their work processes and the capacity of workers to resist hazardous demands from management. ${ }^{12}$ Senior staff can model behaviour that does not promote presenteeism ${ }^{9}$ - for example, by discouraging over-commitment to work and encouraging workers to allow sufficient recovery time from sickness, which may require provision of back-up, particularly in the caring occupations. ${ }^{10}$ Workers with poor health should receive special attention to avoid presenteeism because they are likely to have fewer resources to call on to resist its negative impact. 
Occupational physicians should avoid "standardised" responses to return to work. The worker must be placed within the workplace context and the influences on absenteeism and presenteeism in that workplace, and how the person responds to them. ${ }^{3}$ Manual labourers and clerical workers may have to be discouraged from attending work for different kinds of medical conditions. For example, some musculoskeletal problems may be more problematic for one group than for the other.

Occupational physicians should consider developing indices of workplace presenteeism that would help them when advising both employers and employees. Managers and occupational physicians need to be alert to the findings that even though presenteeism may have some positive effects in the short term-particularly for healthy workers with high job satisfaction-it is likely to be negative in the long term. ${ }^{9}$ Workers with chronic conditions, who by some definitions will always be sick at work, provide an important contrasting consideration. However, evidence suggests that for many the pressure to work when sick is intense, and more effort is needed to prevent this health debilitating behaviour.

Competing interests: All authors have completed the ICMJE uniform disclosure form at www.icmje.org/coi_disclosure.pdf (available on request from the corresponding author) and declare: no support from any organisation for the submitted work; no financial relationships with any organisations that might have an interest in the submitted work in the previous three years; no other relationships or activities that could appear to have influenced the submitted work.

Provenance and peer review: Commissioned; not externally peer reviewed.

1 Rantanen I, Tuominen R. Relative magnitude of presenteeism and absenteeism and work-related factors affecting them among health care professionals. Int Arch Occup Environ Health 2011;84:225-30.

2 Aronsson G, Gustafsson K, Dallner M. Sick but yet at work. An empirical study of sickness presenteeism. J Epidemiol Community Health 2000;54:502-9.

3 Johns G. Presenteeism in the workplace: a review and research agenda. J Organizational Behav 2010;31:519-42.

4 Dew K, Keefe V, Small K. "Choosing" to work when sick: workplace presenteeism. Social Sci Med 2005;60:2273-82.

5 McKevitt C, Morgan M, Dundas R, Holland W. Sickness absence and "working through" illness: a comparison of two professional groups. J Public Health Med 1997:19:295-300.

6 Aronsson G, Gustafsson K. Sickness presenteeism: prevalence, attendance-pressure factors, and an outline of a model for research. J Occup Environ Med 2005;47:958-66.

7 Jena A, Baldwin DW, Daugherty S, Meltzer D. Presenteeism among resident physicians. JAMA 2010;34:1166-7.

8 Grinyer A, Singleton V. Sickness absence as risk-taking behaviour: a study of organisational and cultural factors in the public sector. Health Risk Society 2000;2:7-21.

9 Demerouti E, Le Blanc P, Bakker A, Schaufeli W, Hox J. Present but sick: a three wave study on job demands, presenteeism and burnout. Career Dev Int 2009;14:50-68.

10 Löve J, Grimby-Ekman A, Eklöf M, Hagberg M, Dellve L. "Pushing oneself too hard": performance-based self esteem as a predictor of sickness presenteeism among young adult women and men-a cohort study. J Occup Environ Med 2010;52:603-9.

11 Kivimäki M. Working while ill as a risk factor for serious coronary events: the Whitehall II Study. Am J Public Health 2005;95:98-102.

12 Dew K, Taupo K. The moral regulation of the workplace: presenteeism and public health Sociol Health IIIness 2009;31:994-1010.

Cite this as: BMJ 2011:341:d3446 